UDC 513.72

SCOPUS CODE 1802

https://doi.org/10.36073/1512-0996-2021-1-106-118

\title{
Dynamics of the Dual Relationships
}

Sergo Dadunashvili

\author{
Department of Electrical Engineering and Electronics, Georgian Technical University, \\ Georgia, 0160, Tbilisi, Kostava Str. 75 \\ E-mail: dadu@gtu.ge
}

\section{Reviewers:}

G. Dgebuadze, Professor, Faculty of Power Engineering and Telecommunication, GTU

E-mail: project7@gtu.ge

K. Kamkamidze, Professor, Faculty of Informatics and Computer systems, GTU

E-mail: kkamkamidze@yahoo.com

\begin{abstract}
Nature creates its systems in the form of a sequence of hierarchy levels that unite many heterogeneous objects, based on dual relationships.

The article discusses the general principles of work and the manifestation of dual relations, as well as a measuring instrument for their functioning.
\end{abstract}

Key words: duality; manifestation; measurement; operators; relationship; structure.

\section{Introduction}

There are many viruses around a man, and they are much older than humankind is. It often happens that viruses are looking for a new field of activity and become dangerous for man. When a virus changes its natural habitat, crosses the interspecies barrier, and finds a man, many problems and risks arise. Why do cells show philia and love and begin to reproduce a virus? This is how duality works. The cell has its own genome. It acts as a scanner. If a fragment of specified external genetic material gets into the genome's area, the cell will try to embed it into its genome. After all, if she survives, she may be more perfect. For example, a cell can process some chemical element that has not yet been absorbed, produce a new enzyme, and so on. When viruses embed their genetic material into the genome of host cells, the cell may acquire some new qualities, or not acquire, and even die. Nevertheless, cells always try to seize the genetic material they meet so that a more updated and perfect genome is transmitted to the offspring, which provides program-genetic control to her existence. Consideration of the described phenomena as dynamics of the dual relationship <virus (V) |cell (C)> allows obtaining a number of new results.

\section{Main part}

\section{Operators}

The main operators that regulate the whole life process of the universal object $\langle\mathrm{V}| \mathrm{C}>$ are their application, assembly, multiplication and replication.

The application consists in superimposing new generations of viruses and cells on old ones, which in the temporal and spatial perspective takes on the character 
of an innovative-routine evolutionary stratification. The application creates the basis for the progress and continuity of the generations of viruses and cells that are born in dual relations in various organisms in a changing environment. The application obeys the law of additivity. The results of the application can take curly multicolor forms, in particular for viruses, these are their crystalline states.

Assembling - mixing elements from one set.

Multiplication is about maintaining a variety of environments and resources for generic objects. Viruses travel through space and time to reach the cells that target them. The multiplication operators provide an increase in transport, communication, structural and functional diversity for both the environment and resources. Special instruments (multipliers) realize the quantitative distribution and amplification of the transmitted impact.

Replication consists in renewal, repetition of elements of the universal object $\langle\mathrm{V} \mid \mathrm{C}\rangle$, the meaning of which is to ensure accurate copying of hereditary information and its transmission from generation to generation. Replication, as well as other important communication processes in $<\mathrm{V}|\mathrm{C}\rangle$ - transcription and translation, is based on the matrix principle of synthesis and complementary interaction between pairs. All hereditary information necessary for growth, development, metabolism and reproduction is transmitted from the original to the copies in the form of the source code of their existence. In replication constantly ongoing evolutionary process of local innovations and interventions, as well as subsequent routines and conventions. Through the variability of the structure and functions of universal objects, the irreversibility of the time and space of life is realized.

The life cycle of operators of dual relations (DR), presupposes the number and forms of the combination of the main endogenous factors. It is preserved from generation to generation, while maintaining two types of division: one for the material formation of the "body" of viruses and cells of this generation in the process of normal reproduction (through quantitative growth), the other - through qualitative development, for the formation of carriers of their innovative generations. The factorial and resource capabilities of a DR determine both the level of its development and its ability to create its own existence in a changing environment.

All nature is alive, both in whole and in parts. Beneficial and hostile influences come from all directions. Nothing is inactive, but, however, all actions and interactions of both things and beings are based on an active, sympathetic affinity within. Sympathy is a link to the previous one, its source is "engagement". "Engagement" leads to imitation, which is a condition for saving energy and resources. The versatility of imitation is also manifested in the effect of similarity, when similar objects experience similar effects. Breaking the chain of "engagement" leads to the collapse of DR.

In the integrity of the DR, opposites are complementary. The principle of complementarity is when two or more quantities together form one whole. The whole can be expressed by the sum of complementary parts or by their multiplication. For example, the energies of the two poles are additional, in total they will make up the total energy of the whole: $A_{1}+A_{2}=A=$ Const. By increasing the energy of one plus, we automatically reduce the energy of another by the same amount. The same entity demonstrates a different type of additional dependence in the interaction. The pair is made by the resource of the $A_{1}$ and the action $F_{1}$. Their mmultiplication (act $\gamma$ ), is a constant value for a given completely: $\gamma=A_{1} \cdot F_{1}=$ Const. At the other pole of the whole, as a result of the similarity, there is a second unit with an inverse ratio of resource and action, but with the same constant: $\gamma=\mathrm{A}_{2} \cdot \mathrm{F}_{2}=$ Const.

The first kind of complementarity is applicable to variables that are measured by the same measure. Such quantities are called additive. Variables related to the second kind of complementarity are multiplicative, qualitatively different, and it is not the sum that remains constant, but the composition. In mechanical space, additive and multiplicative complementarities look like completely different patterns. However, they are translated one into another by the operation of logarithm or potentiation, which confirms their "relationship" at the next level of reality.

The principle of complementarity is a fundamental law of universal significance in Nature. Ultimately, 
similar restrictions in the interdependence of two quantities exist between states that are inextricably linked, but at the same time incompatible and are forced to replace each other in time. The duality of opposites of inseparable things and events, permeates all life processes. The fundamental nature of dual relations DR, universality and continuity in the existence of dual forms, their regularities of limitation and isolation, manifestations of their symmetry and asymmetry, characterize different facets of this fundamental essence.

In reality, the dual relations are a universal integral object (UO), the properties of which are unique. This is the basic essence that serves as a "matrix" with which "prints" are made in any spheres of existing, and thanks to this, everything is created according to a single plan. $\mathrm{UO}$ is the creator of all that is and carries its meaning. This is the proto-intelligence of all things, dispersed throughout the Existence. The form-forming individual principle determines the ubiquitous intelligent invariant, which carries the meaning of each object of Nature. Therefore, for the analysis, the ideal UO is taken, which manifests itself at any levels of the hierarchy.

From the point of view of the laws of limitedness and isolation, DR has a limited number of permissible states at each level of the hierarchy, which places UO within the boundaries of normativity. DR between the two parts of the UO completely affects both sides and concerns all areas of their activities in which duality manifests itself. In DR, the content of each part can change from simple to complex. As a result of sequential complication, there is a transition to the level of its ultimate complexity (level 1). The process of change can also go in the opposite direction, "from the complex to the simple." Because of consistent simplification, a transition to the level of proto-Essence occurs (level 0).

\section{Dual Relationship Structure}

Dual relationship (DR) is the vital axis of existing. The interrelation of parts DR is characterized by the unity and mutual complementarity of their boundaries. The inner unity of two mutually complementary parts characterizes the limit that reflects the intelligent design of creation. Every time when the formation of an integral boundary is completed, it is renormalized. The measure of the border is always outside the considered DR and carries restrictions on the limits of change in the values of the DR.

Any relation has its own measure and its own designated direction in "space-time". The measure ensures the self-sufficiency of the relationship. The object type is already set at the zero level. The levels in relation are balanced relative to each other and form the "instrument of assessment" of the (DR). Each level, in relation to the adjacent one, is characterized by a negative dimensionality of relations. This algorithm reflects the principles of forming boundaries at all levels of organization of DR.

The Unified Formula of the Unified Law $(\Phi)$ of DR is based on the fundamental concept of a universal object $\{\mathrm{UO}\}=<\mathrm{X}_{1} \mid \mathrm{X}_{2}>$, which consists of two parts: $\mathrm{X}_{1}$ and $\mathrm{X}_{2}$. Symbol "|" means DR is being investigated. To indicate the state of the relationship, angle brackets of the form $<\mathrm{X}_{1} \mid \mathrm{X}_{2}>$ for the statics of the relationship and $>\mathrm{X}_{1} \mid \mathrm{X}_{2}<$ for the dynamics of the relationship are used.

UO does not carry a "subject aspect", the subject of research is not Objects and (or) Subjects, but the relationship between them. All DR of any nature have common basic properties. These properties are characterized as "external" and "internal". In DR with external duality $(\uparrow)$, two of its mutually complementary aspects are observed at once. This attitude is called "manifested." DR with internal duality $(\downarrow)$ characterizes the unity of the external and internal aspects of the dual relationship. This attitude is called "un manifest".

In DR with external duality, the category "Measure" reflects the degree of balance of the elements of the relationship. It characterizes the measure of the interpenetration of the parts of the dual relationship into each other. Sense DR with internal duality is determined by the external border of the "final limit" serving as a unit measure of internal relationship. Because of this, DR with internal duality is normalized and is characterized by a complete balance of the relationship as a whole. For such DO's, the number one is the largest quantitative estimate.

Two procedures represent the natural operating mechanisms in DR. Reflection and mapping of the subset $X_{1}$ into the subset $X_{2}$ are the rules according to which 
each element of the subset $X_{1}$ is associated with an element of the subset $\mathrm{X}_{2}$.

Because of the operation of reflecting the corresponding external duality, the object changes its appearance, which is analytically fixed by a change in sign:

$$
\phi: \mathrm{X}_{1} \rightarrow \mathrm{X}_{2} \text { and } \mathrm{X}_{1}=-\mathrm{X}_{2}
$$

As a result of the display operations the corresponding internal duality, the object is reborn and receives an otherwise existence.

$$
\psi: \mathrm{X} \rightarrow \mathrm{Y} \text { and } \mathrm{X}=\mathrm{Y}^{-1}
$$

The mapping that assigns to each element $(Y)$ its preimage $\psi^{-1}: Y \rightarrow X$ is called inverse for the mapping $\psi$. Balance DR and the integrity of the object, suggests that between the poles of internal duality there is an inversely proportional relationship of the form:

$$
\frac{X}{1}=-\frac{1}{Y} \text { and } \frac{Y}{1}=-\frac{1}{X}
$$

Those "turned inside out" " $\mathrm{Y}$ " is reborn into " $\mathrm{X}$ ", and "X" "turned inside out" becomes like "Y". This is how the levels of existence and otherwise existence are connected. At the same time, DR is not violated; it becomes external, and acquires other, mutually complementary properties:

$$
(+\mathrm{X})=(-\mathrm{X}) \text { and }(+\mathrm{Y})=(-\mathrm{Y})
$$

The minus sign in these expressions determines not the equality of positive and negative numerical values, but their poise balance.

Formula $(\Phi)$ of Evaluation of the Participants of the Relationship (1) can be used to determine the quantitative and qualitative Measures of the balance of any objects, regardless of their nature. This formula allows to give a qualitative assessment of the essential and functional aspects of UO. However, with such an "assessment" (DR), the concepts of "identity" and "equality" may differ from the generally accepted ones. They have a deeper meaning, reflecting the laws of conservation of symmetry DR.

\section{Principles of Dual Relationship's Conservation}

Any phenomenon in Nature and in society has not only two extreme discrete static manifestations of the "better-worse" type, but also four dynamic manifestations in two pairs of qualitative-quantitative asymmetries. They clearly and definitely reflect the unity of level and measure and show their complementarity "in IMAGE and LIKENESS".

The laws of preservation of symmetry in a dual sense reveal the principle of operation of its natural operating mechanisms. DR with external and internal duality are characterized by invariant transformations.

The law of conservation of dissymmetric of the dual relation ( $\alpha$-invariance) operates at each level: For the variables $\left(\mathrm{x}_{\mathrm{i}}\right)$ at the zero level, the following relation is valid:

$$
<\mathrm{X}_{1}\left|\mathrm{X}_{2}>=-<\mathrm{X}_{2}\right| \mathrm{X}_{1}>
$$

For variables $\left(\mathrm{y}_{\mathrm{j}}\right)$ at level 1 , the following relation is valid:

$$
<\mathrm{y}_{1}\left|\mathrm{y}_{2}\right\rangle=-<\mathrm{y}_{2} \mid \mathrm{y}_{1}>\text {. }
$$

These relations are related as "right" and "left", characterizing the integrity of the external DR. Due to this polarity, when the value of one-pole DR changes, the other pole also changes its value. $\alpha$-invariance is the result of the law of reflection and characterizes of dissymmetric (left becomes right and vice versa, right becomes left).

If we associate the action $\mathrm{F}\left(\mathrm{x}_{1}, \mathrm{x}_{2}\right)$ with the relation $<\mathrm{x} 1 \mid \mathrm{x}_{2}>$, and with the relation $<\mathrm{x}_{2} \mid \mathrm{x}_{1}>$ the action $-\mathrm{F}\left(\mathrm{x}_{2}, \mathrm{x}_{1}\right)$, an additional to action $\mathrm{F}\left(\mathrm{x} 1, \mathrm{x}_{2}\right)$, then obtain a new relation in which the action of objects on each other has the character of interaction:

$$
\operatorname{Fxx}\left(\mathrm{x}_{1}, \mathrm{x} 2\right)=-\mathrm{Fxx}(\mathrm{x} 2, \mathrm{x} 1)
$$

Actions with which objects act on each other are always equal in magnitude, oppositely directed and act along the straight line connecting these objects.

Resource conservation law of the symmetry of the dual relation ( $\beta$-invariance).

The designation $\langle\mathrm{X} \mid \mathrm{Y}\rangle$, already initially characterizes the mutual complementarity of the "poles of the dual inter level relationship", which is characterized by the law of conservation of the symmetry of the exchange of resources (energy, charge):

"What decreases from one "pole" (X or Y) will be added to another "pole" (Y or X) ".

$\beta$-invariance metamorphose "external to internal, and internal to external." Those, the contents of objects and subjects are "turned inside out". This procedure for a dual inter-level relationship, taking into account the 
reciprocity of the corresponding actions, can be written in the following general form:

$$
F_{X Y}(X)=F_{Y X}(Y)^{-1}
$$

This identity means a balance between external and internal actions, balancing the "external" and "internal" environment of objects and subjects of any nature. Here, the expressions on the left and right sides get different dimensionality, reflecting the law of resource inversion (reversibility).

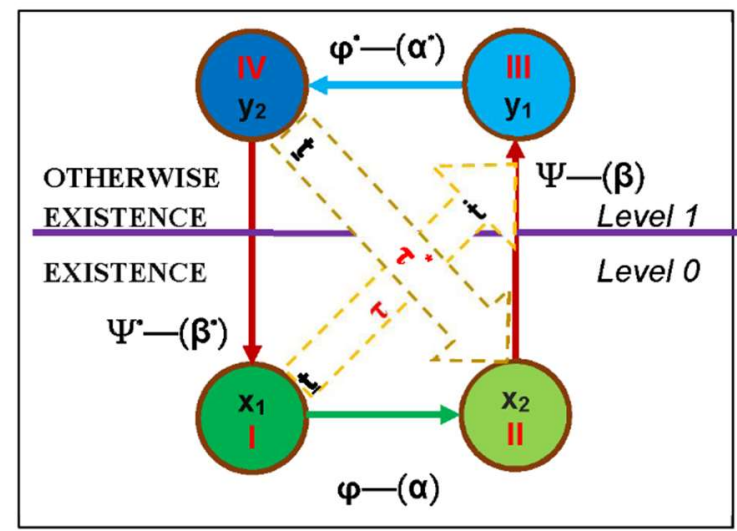

Figure 1 Circuit

Operating mechanisms in a dual relationship represent sense the form of a circuit diagram shown in Fig. 1. Consider the processes occurring in it.

$>\alpha$-invariance characterizes dissymmetric - left becomes right and vice versa, right becomes left. As a result of the reflection operation, the object changes its appearance and gets an idea, which is analytically fixed by changing the sign.

$>\beta$-invariance transforms the external into the internal, and the object receives otherwise existence.

In Figure 1, when "reflected" and "turned out", the pairs change the colors of their components. The uniform color of the shape of the components indicates their common origin.

The law of combined conservation of the symmetry of the (DR) ( $\alpha \beta$-invariance).

If identity (3) is combined with the previous one (2), then the general law of symmetry conservation is obtained, in accordance with which tool of DR works:

$$
F(X)=-(F(Y))^{-1}
$$

On the other hand, expanded $\mathrm{DR}(\mathrm{X} \mid \mathrm{Y})$ and using the variables at the appropriate levels, get:

$$
\text { Reflection }^{-\frac{x_{1}}{x_{2}}}=-\frac{\left(\frac{y_{2}}{y_{1}}\right){ }_{\text {Recovery }}^{-1}}{\bigcup_{\text {R }}}
$$

This is the formula $(\Phi)$ conservation of the DR. According to the obtained expression, works the "assessment tool", which determines the balance of relationships between the poles of any nature DR. Thus, the invariant transformations contents determine the work of "assessment tools" of DR.

The static expression of the Law of conservation of DR for the "assessment tool" reflects the balance of measures (y) and corresponding actions ( $\mathrm{x}$ ), which, according to (5), is written in multiplicative form as:

$$
\left(x_{1} \times y_{2}\right)=-\left(x_{2} \times y_{1}\right)
$$

The law of the tool - "lose in action - win in resources, win in action - lose in resources", manifests itself in full at all stages of activity of DR. In the instrument of $\mathrm{DR}$, balance is achieved by compensating for various measures by finding the corresponding positions with adequate resources.

Whenever there is an equilibrium relationship between the left and right sides of the instrument:

$$
\left[\frac{F(X)}{1}\right]^{+1}=-\left[\frac{1}{F(Y)}\right]^{+1}
$$

action is normalized, that is, they are characterized by a single measure:

$$
F(X) \times[F(Y)]^{-1}=-1
$$

The universal law of conservation the symmetry of the dual relation ( $\alpha \beta \tau$-invariance) is obtained by replacing in the above formula (6) the "unit" relation by the "systemic" one:

$$
\left[\frac{F(X)}{\boldsymbol{\tau}\left(t_{0}, t\right)}\right]^{+1}=-\left[\frac{\boldsymbol{\tau}\left(t, t_{0}\right)}{F(Y)}\right]^{+1}
$$

Time $(\tau)$ is used as a Measure. Already from the meaning of the dual category "Measure" it follows that the category "Time" is also characterized by duality and mutual complementarity. In expression (7), on the left side, time is directed "outside", and on the right side - it is directed "inward". 
The formula $(\Phi)$ explains the interconversion of the "four elements" during rotating invariant transformations in the circuit (see Fig. 1) and is written as the following equality:

$$
\frac{\left[\left(\frac{\alpha}{\beta}\right)^{+1}=-\left(\frac{\beta^{*}}{\alpha^{*}}\right)^{-1}\right]}{\tau}=-\frac{\tau^{*}}{\left[\left(\frac{\alpha^{*}}{\beta^{*}}\right)^{+1}=-\left(\frac{\beta}{\alpha}\right)^{-1}\right]}
$$

This formula reflects the statics of equilibrium of conservation laws at $\tau=\tau^{*}$.

The unified law of DR indicates that as long as the duality persists, the symmetry conservation law $(\alpha \beta \tau$ invariance) DR is not violated. They move from one form of conservation to another.

In this Equation, time on the left side flows from the "Past $(\mathfrak{t})$ " through the Present $(\tau)$ to the "Future $(\mathfrak{t})$ ", and on the right side, on the contrary, from the "Future $(\mathbf{t})$ " through the Present $\left(\tau^{*}\right)$, in the "Past $(\mathfrak{t})$ ". This means that the time on the left and right sides is reversible in relation to each other. It plays the role of a Measure and determines the direction of self-regulation processes. The formula expresses the unity of the two levels and the meaning of the duality of time. Integrity of $\mathrm{UO}$ is characterized by the unity and complementarity of its two levels. The meaning of this postulate and the order of activity DR are illustrated in a rotating circuit in Figure 1.

The manifestation of each species DR has a Single Measure and a Single intelligent design, imprinted on each specimen of the species. The intelligent design of the future creation is stored in the unmanifest DR, for all other components DR are determined in fractions of the unit by which it is evaluated. This is the most important property DR with internal duality.

A measure modulo can have three values. If the Measure is equal to one, then the border of DR is completely balanced with its own intelligent design and is stable. In other cases, DR will either disintegrate or transform into a more complex formation. If the balance is disturbed, the balancing process begins.

For the occurrence case:

$$
\operatorname{Measure}(\mathrm{M})=\left|\frac{\tau}{\tau^{*}}\right|^{+1}>1
$$

at using the resource, optimal self-regulation of the relationship between the four "elements" will follow the expression:

$$
\begin{gathered}
\frac{\left[\left(\frac{\alpha}{\beta \rightarrow \max }\right)^{+1}=-\left(\frac{\beta^{*} \rightarrow \max }{\alpha^{*}}\right)^{-1}\right]}{\tau} \\
=-\frac{\tau^{*}}{\left[\left(\frac{\alpha^{*}}{\beta^{*} \rightarrow \min }\right)^{+1}=-\left(\frac{\beta \rightarrow \min }{\alpha}\right)^{-1}\right]}
\end{gathered}
$$

This expression characterizes the process of transformation of the intelligent design into a formalized materialized object.

For the case:

$$
\operatorname{Measure}(\mathrm{M})=\left|\frac{\tau}{\tau^{*}}\right|^{+1}<1
$$

the reverse process of dematerializing the relationship between the four "elements" follows another expression:

$$
\begin{aligned}
& \underline{\left[\left(\frac{\boldsymbol{\alpha}}{\boldsymbol{\beta} \rightarrow \min }\right)^{+1}=-\left(\frac{\boldsymbol{\beta}^{*} \rightarrow \min }{\boldsymbol{\alpha}^{*}}\right)^{-1}\right]} \\
& =-\frac{\boldsymbol{\tau}}{\left[\left(\frac{\boldsymbol{\alpha}^{*}}{\boldsymbol{\boldsymbol { \beta }}^{*} \rightarrow \max }\right)^{+1}=-\left(\frac{\boldsymbol{\beta} \rightarrow \max }{\boldsymbol{\alpha}}\right)^{-1}\right]}
\end{aligned}
$$

In this expression, forward and backward time is not balanced, and the "excited" circuit generates changes in the proportions of the four "elements" that determine a new stable state. The formula directly shows the situation in which a violation of the conservation law can take place: a violation of the absolute conservation law DR can occur when UO goes beyond its own systemic (time) limits of existence.

The patterns described above reflect the initial four "elements", the four foundations of the source code of Being UO, generating their own "space-time". But the fourth "element" is not necessarily time. Other system variables of any nature can be used as it. The fourth "element" reflects the "measure" and "systemic meaning" of the 3-dimensional space of existence. During the invariant transition to the "other space", the self- correction of its own "content", "goal" and "measure" takes place. 


\section{Dual Relationship Manifestations}

The dual relationship (DR) of reality forms can be reduced to the following types:

"Object-Object", "Subject-Subject", "Object-Subject".

"Object-Object" and "Subject-Subject" represent relationships with external duality. "Object-Subject" represents a relationship with internal duality. The relationship between the Object and the Subject is that an object with a structured resource manifest it explicitly. The subject, being structureless, carries a message (offers) about this resource. This is how the coherence and unity of the structural and functional aspects of the relationship is manifested.

Four components of DR are:

$>\operatorname{OBJECT}\left(\mathrm{x}_{1}\right)$ is a part of objective reality $\{\mathrm{X}\}$.

$>$ SUBJECT $\left(\mathrm{x}_{2}\right)$ is a physical image that coincides with its content category, the likeness of an object. The Subject expression carries all the properties of the real object, but is not the original Object.

$>$ IDEAL IMAGE (y2) is the Measure of the Object in the subjective reality $\{\mathrm{Y}\}$. The image is in another being and is otherwise existence with an independent content - an intelligent design. It is a pure subject of natural idealism.

> IDEAL IMAGE (y2) is the Measure of the Object in the subjective reality $\{\mathrm{Y}\}$. The image is in otherwise existence, with an independent content - an intelligent design. It is a pure subject of natural idealism.

> LIKENESS IMAGE $\left(\mathrm{y}_{1}\right)$ - these are congenital properties of an object, representing a memory (seed) in which all previous revivals and all future offspring of the object are stored.

The highlighted components are key. They represent different poles of one holistic UO. The essence of these four components is revealed using the following scheme (Fig. 2). The diagram shows the real work processes of DR $<$ Object $\mid$ Subject $>$.

"0" -level - Substantial reality. Variety of corporeality. Physical actions and their limits.

"1" -level - Ideal actions, work - individual and social thinking. The rational latent beginning as intelligent that knows about the "0" - level.
In the (Fig. 2), level 0 corresponds to objective reality. The concept of "Measures" represents the relationship between the two poles of subjective reality at level 1. Manifestation "by Image and Likeness" corresponds to the Law of Evolution DR and reflect the principles of natural operationalization. The figure shows the content operands DR - FXY and Fyx: there can be no object $(\mathrm{x} 1)$ without the image of the object $(\mathrm{y} 2)$ and likeness of the image (y1) without the subject (x2).

If the external relation $\left\langle\mathrm{x}_{1} \uparrow \mathrm{x}_{2}>\right.$ at level 0 is identified with the evolution of the Object $\left(F_{12}\right)$, and the external relation at level $1<\mathrm{y}_{1} \uparrow \mathrm{y}_{2}>$ is identified with the work of the Subject $\left(F_{21}\right)$, then the relationship in the "assessment tool" evaluating the relation <Object|Subject> will be represented by an identity:

$$
\left(\frac{\text { Object }}{\text { Measure of object }}\right)^{+1}=-\left(\frac{\text { Measure of subject }}{\text { Subject }}\right)^{-1}
$$

Identity is composed in such a way that nothing can be rearranged without violating the content of DR and the content of proportion.

Algorithm is an instruction that includes a certain order of final actions performed to solve the problem. Algorithm - a scheme for implementing an idea. Program is a record of a sequence of instructions for realization of an idea by concrete tools - instrumentality.

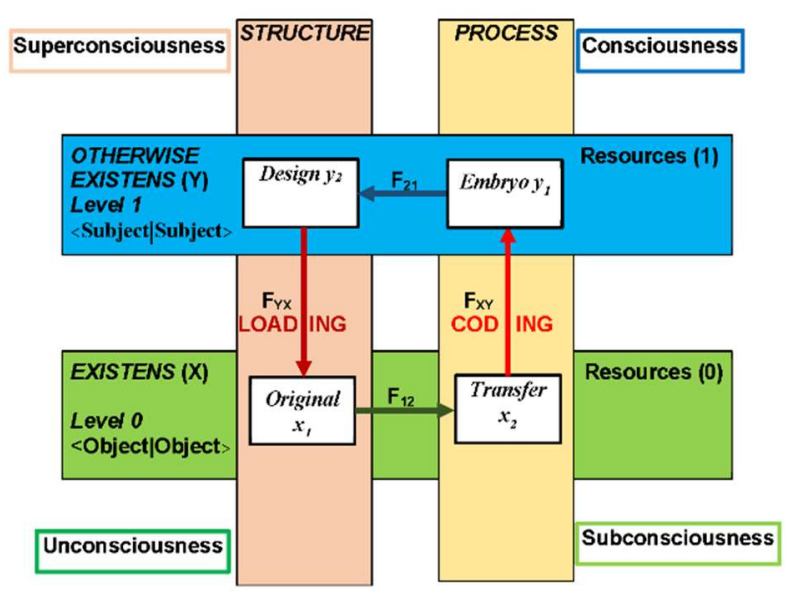

Figure 2. DR <Object $\mid$ Subject $>$ 
The diagram in Fig. 2 shows the similarity of the forms of direct and inverse proportions forming the channels of penetration to levels 0 and 1 . However, these proportions are ambiguous and have different meanings. The measure of transformation of the external relation $<\mathrm{x}_{1} \uparrow \mathrm{x}_{2}>$ at level 0 shows the degree of balance between the Object and the Subject. If this Measure is not a single one, then it determines distortions in the relationship of the <Subject-Object> pair. These distortions will be present at all levels of materialization of the intelligent design, giving rise to defects in the created object.

A similar transformation of the external relation $\left.<\mathrm{y}_{1} \uparrow \mathrm{y}_{2}\right\rangle$ will also occur at level 1 of the scheme, when the pair < Measure of object ( $\left.\mathrm{y}_{2}\right)$ - Measure of subject $\left(\mathrm{y}_{1}\right)>$ functions. The measure of balance always stands outside the considered dual relationship, performing the functional role of regulation, control and management of evolutionary processes. It determines the area of the relationship < Object $\left(\mathrm{x}_{1}\right) \mid$ Subject $\left(\mathrm{x}_{2}\right)>$ and characterizes its system parameters. At the same time, DR determines the content, the goal, and the measure of the process.

The essence of subjective reality is INFORMATION (I), which evaluates the ideal image of an OBJECT. Information, carrying the properties of an Object, is not an Object itself. It is located outside its poles and characterizes the degree of their balance and the laws of reflection and reflection between them. The function of information is to be a measure of the manifestation of these poles. The category "Measure", being universal, is applicable to objects of any nature and is expressed by information (I). "Incorporeal" DR, which is defined as information, is used at measurement "conversion rate" of an Object into a Subject and vice versa. If the outcome is unity, there is a complete and adequate picture of balance between the Object and the Subject. Otherwise, the phenomenon of "information defect" arises. The main principles that are used in such measurements are the laws of conservation of these relations.

On the one hand, information characterizes a real Object that manifests its material properties. Information, carrying the properties of this object, thereby becoming an object of information. On the other hand, it acts as the subject of information, coding the reflected by means of the laws of display. Accordingly, information exists in two forms - manifested information (Information Object) and non-manifested (encoded) information created by a functioning information subject.

The content of the "primordially" basic code words, represents a beginning that never existed, but which always exists as a "beginning", as a "principle". The "primordial" is by no means the past, but rather the future. It is an active sympathetic means from within and is not comprehended as a meaning, but it is built into and inserted into the internal order of its alphabet.

Information is used in two ways, both for the materialization of the Object, and for its dematerialization (encoding). Dematerialization characterizes the process of regeneration of the Information Object into the Information Subject (into the seed), and then its reflection into the Image (the intention of the future Creation). The reverse process of materialization of an Object - creation, comes from the Image, which carries its intelligent design within itself. Thus, in subjective reality, a feedback loop (FB) is formed, due to which the processes of materialization and coding in DR are stabilized.

The nature of the processes occurring in DR reveals the meaning of the concepts of materialization and coding. For a specific external relationship $<$ Object $\uparrow$ Subject $>$, based on (8), obtain expression is:

$$
\frac{x_{1}}{x_{2}}=-\left(\frac{y_{2}}{y_{1}}\right)^{-1}=\mathbf{I}
$$

Relationship between the manifested Information Object and the Information Subject show in Fig. 3. The measure of the formed FB chain includes the measure of materialization and the measure of coding. It characterizes the processes of balancing and stabilization in an assessment tool that manifests the fundamental role of Information. Information acts as a catalyst for the relationship between the Object and the Subject of information within a certain quality, fixed in the contour $<\left(\mathrm{x}_{1} \rightarrow \mathrm{x}_{2}\right) \mid\left(\mathrm{y}_{1} \rightarrow \mathrm{y}_{2}\right)>$. In this case, the conversion of Information (materialization, dematerialization) is carried out in accordance with the laws of conservation. 


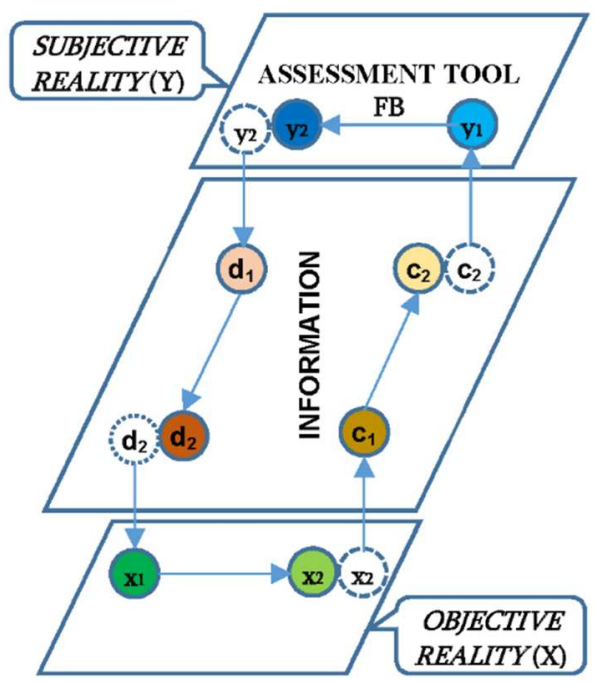

Figure 3. "Incorporeal" DR

In figure 3 intermediate modes (not yet and no longer) are designated by a dotted circle.

- $\alpha$-invariance leads to the fact that at the level "X", the object becomes no longer $\left(\mathrm{x}_{1}\right)$, but its mirroring ( $\left.\mathrm{x}_{2}\right)$, is not yet " $\mathrm{Y}$ ".

- $\beta$-invariance maps " $\mathrm{x}^{\prime}$ " to $\left(\mathrm{y}_{1}\right)$, for this the object is transferred to an intermediate information level, subjected to coding "C", and it is no longer "X", after which it gets to the level "Y".

- $\alpha^{*}$-invariance leads to a mirroring (y1) in (y2), that is, the object becomes further like (Y).

* $\quad \beta^{*}$-invariance maps "yz" (Y) to "X" ( 11$)$. First (y2) gets to the intermediate information level, that is, the object is no longer " $\mathrm{Y}$ ", decoded by " $\mathrm{D}$ " and returned to being.

The most important thing is beginning to keep this "not yet" ("almost"). From "almost" to "already" there is sometimes an insurmountable distance during life (in time and/or in space).

The dimension of the Measure is not directly "manifested" however, it carries limitations on the limits of change in the values of the $\mathrm{DR}$ and can change the direction of traversing the contour to the opposite. The closed loop forms the infrastructure conducts the evolution of the intelligence. At the same time, the four elements are manifested at all levels of the hierarchy of the intelligence and their relationships correspond to the laws of conservation symmetry. The embeddedness of an object in a contour indicates that intelligence and matter are interconnected, one exists only for the other, both appear and disappear together, and one is only a reflection of the other. In fact, they represent the same entity viewed from different points of view. The consideration includes not only the subject, but also space, time and causality, which depend on how the subject is defined.

The subject's consciousness is manifested when it interacts with objective reality through information. Information, as a functioning subject of relations, is a mediator with objective reality. Consciousness manifests itself in work and at the end of which it disappears. As a result of work, an image of an object is formed and shown to the subject.

Consciousness manifests itself as a unity of its structural and functional aspects. The structural aspect of consciousness reflects events adequate to it, which are perceived at the level of sensations. The functional aspect of consciousness reflects certain changes associated with each event. The principle of long-range action means a resonant exchange mode, in which distances and time are rolled up into a unit.

The rotating contour manifests consciousness in the four elements. The manifestations of consciousness in the circuit occur in accordance with the laws of conservation. Consciousness without an object is not consciousness at all. If the attributes of non-local and local behavior are used in the description of consciousness, then reflection, long-range action and other effects that look anomalous from the point of view of locality are possible for "non-local" consciousness. The mind without consciousness loses these properties [1].

Consciousness can bring information into the surrounding reality by spreading the results of disclosure and cognition of relationships. Everything social belongs to the area of "consciousness", which is much broader than the realm of the social itself. These are not spontaneous processes; consciousness has its own Measure, which completely determines the direction of the evolution of life.

Knowledge is a real-life resource. This resource is the 
life-giving core of the "four elements". Knowledge is transformed into the Unified Knowledge (Unit), which then goes again to "be fruitful and multiply". "Rotation" in the contour and the process of cyclic renormalization of Knowledge are determined by the Unified law of evolution of "space-time".

The unconscious is the most important factor in existence and represents a powerful force that opposes consciousness. It is beyond consciousness. Among the unconscious drives, there is also an innate tendency to destruction and aggression, which finds its ultimate expression in the "death instinct" opposed to the "life instinct".

The subconscious mind operates with local sensations, emotions and feelings. This is the units of the program by which the consciousness operates.

Super consciousness is the ideals of all kinds and types of life, "crystallized" ideals and norms of society. The supra-individual "general significance" in the individual is the result and expression of his psychophysiological organization.

The categories sub consciousness $(\mathrm{P})$, unconsciousness (B), consciousness $(\mathrm{C})$ and super consciousness $(\mathrm{N})$ represent four complementary "elements" of the information space, which speak different languages.

\section{Assessment Tool of Dual Relationship}

Dual relationship (DR) is the universal encoder for everything. The ideal sign character $\{0 ; 1\}$ represents the alphabet of exactly the "code" that is ubiquitous. Omnipresence and unity do not mean uniformity. A universal object (UO), like an encoder, stands at the crossroads of all paths along which the processes of materialization and dematerialization take place. It has an adequate mechanism for carrying out such transformations and rebirths. Its alphabet $\{0 ; 1\}$ is always and everywhere applicable to both the "input" and "output" of elements. Since the duality is the most fundamental regularity of existence and is reflected in properties of DR, its Law characterizes changes in the properties of any self-sufficient DR, regardless of its nature.

The systemic properties of the assessment tool are manifested when, as a result of the "closure" of the "output y2" of this circuit to the "input $x 1$ ", an am- phiphilic unification of levels occurs. The process of materialization begins and holistic object, with the unity of structure and function, appears. By closing the "output" of the last element to the "input" of the first, the dual relation leads to periodic variability DR, generates a closed cycle of transformation of this ratio and its cyclic renormalization.

Only after completing a circumvolution in the loop, at the moment of "binding" of the Last element to the First, there is return to level 0 , to the source of the cyclically repeating "rotating" process. The initial element at level 0 , being controlled from level 1 , generates a qualitatively new object in each period. When the output of the last element becomes the input of the first, then at this moment, the synthesis of a more complex structural form is carried out and a new function is created.

The unity of the essential (structural, discontinuous, local) and functional (structureless, continuous, nonlocal) aspects of matter and the multilevel formation of this unity allows us to reveal the nature of the closure of the essential beginning (root cause) at the end (consequence of consequences).The unity of discontinuous and continuous spaces characterizes the duality of any proper space. Therefore, the initial DR of any proper space can rightfully be called relationship <local|nonlocal $>$. The local space defines the Information Object, and the nonlocal space is already the Information Subject. Information is always dual and carries in itself the memory of both the Information Object and the Information Subject. This is that otherwise existence in which matter disappears and from which it is reborn.

The assessment tool contains intelligent design of the formula $(\Phi)$ of existence life DR. Any DR is "measured and balanced" by the assessment tool and described by the Formula $(\Phi)$. The instrument consists of two parts, which are related to each other as "external" and "internal" [2]. These parts are proportional to each other and balanced relative to each other in the process of objective and systemic interaction (see Fig. 4). The formula $(\Phi)$ reflects not the functional dependencies between the four components of the instrument, but the operations of balancing the relationship between them. The tool characterizes the universal law of conservation of resource. 


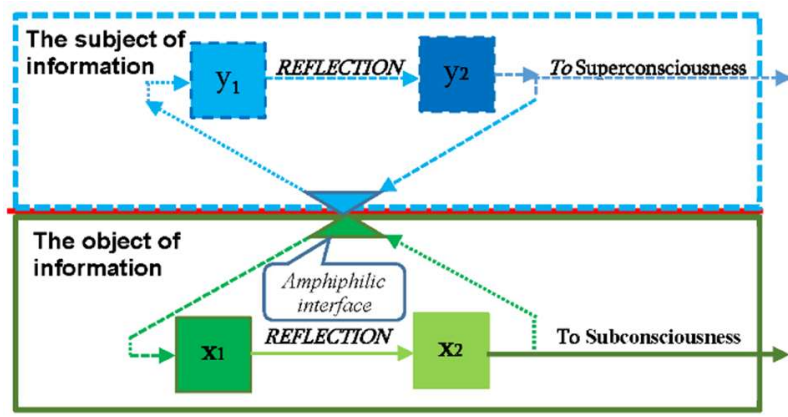

Figure 4. Assessment Tool

Block diagram shows an integral two-level object $\left.\{\mathrm{O}\}=<\mathrm{X}|\mathrm{Y}\rangle=<\left[<\mathrm{x}_{1}\left|\mathrm{x}_{2}\right\rangle \mid\left\langle\mathrm{y}_{1} \mid \mathrm{y}_{2}\right\rangle\right]\right\rangle$. The tool has crossfeedback (FB) that provides the process: "Last (y2) becomes first $(\mathrm{x} 1)^{\prime \prime}$.

\section{Conclusion}

Information is a measure characterizing the "conversion rate" in relation to $\langle\mathrm{X} \mid \mathrm{Y}\rangle$. From the physical side, it appears as an object of information ( $\left.x_{2}\right)$. In otherbeing, it acts as the subject of information ( $\left.\mathrm{y}_{1}\right)$. Objects and subjects of information form an information space. As a result of their activity, space is formed with its own alphabet of symbols and its own single language. Objects of information participate in this process as imprints of physical signals of the original object $\{\mathrm{O}\}$. They are similar to the original object, and are its symbolic representation and define the alphabet of the information space. Information subjects participate in this process as derivatives of the original alphabet, which define the words and language of the information space, based on which composition is created, in the form of a similarity of the likeness image ( $\left.\mathrm{y}_{1}\right)$ and ideal image (y2) of the original object $\{\mathrm{O}\}$.

The principle of complementarity and complementarity operates between the levels of reality. Their mutual mapping occurs. The object's expression is transformed into a physical image representing the likeness of the object. When it is projected to the next level, the physical image is turned inside out and it is dematerialized into a likeness of an image, which, being reflected from the screen of consciousness, is transformed into the ideal image of an object. The image of an object is projected onto the physical level by materialization in an amphiphilic interface and turns into a perceived image of the object. The information objects which are in an objective reality, are put in conformity with information subjects which are in a subjective reality, that is code prints, on the basis of complementary codes of a subjective reality. The corresponding print serves as the code image of original object [3-7].

\section{References}

1. S. Dadunashvili. System Pattern of Consciousness Functioning - International Symposium on Biomedical Engineering and Medical Physics. Riga, Springer, IFMBE Proceedings 38, p. 5-10. (2012). (In English);

2. S. Dadunashvili. System formation models in biosphere and a noosphere. - Tbilisi. Proceedings of the Georgian Technical University, №3 (414), p. 224-232. (1997). (In English);

3. S. Dadunashvili. Bio signals Processing in the Structural Levels of Body - World Congress on Medical Physics and Biomedical Engineering. Beijing, Springer, IFMBE Proceedings Vol. 39, p. 581-584. (2012). (In English);

4. S. Dadunashvili. Becoming of Ubiquitous Sensors for Ubiquitous Healthcare. - World Congress on Medical physics \& biomedical Engineering. Toronto, Springer, IFMBE Proceedings, IUPESM 2015, Pages 1416-1418. (2015). (In English);

5. S. Dadunashvili.. Multilevel Signal Processing for Biomedical Nanodevices. - Springer, IFMBE Proceedings Vol. 55, p. 329-331. (2016), (In English);

6. Bar-On Yinon M., Flarnholz Avi, Phillips Rob, Milo Ron, SARS-CoV-2 (COVID-19) by the numbers. 2020. (https://bit.ly/2WOeN64 ). (In English);

7. S. Dadunashvili. Medical Expert System with the Properties of Artificial Intelligence - World Congress on Medical Physics and Biomedical Engineering. Prague, Springer, IFMBE Proceedings Vol. 68/1, p. 315-319. (2018), (In English). 


\section{UDC 513.72}

SCOPUS CODE 1802

https://doi.org/10.36073/1512-0996-2021-1-106-118

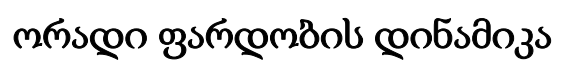

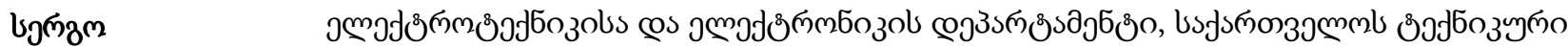

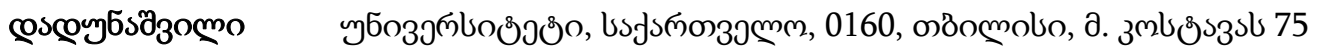

E-mail: dadu@gtu.ge

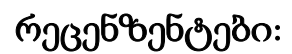

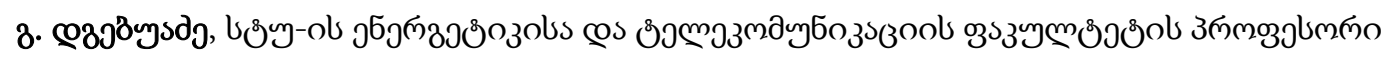

E-mail: project7@gtu.ge

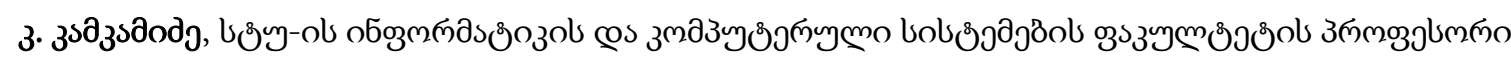

E-mail: kkamkamidze@yahoo.com

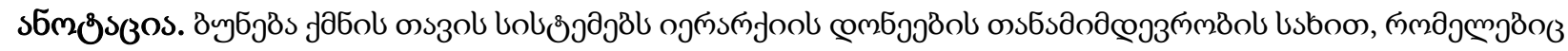

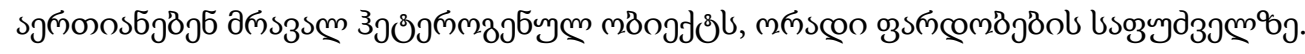

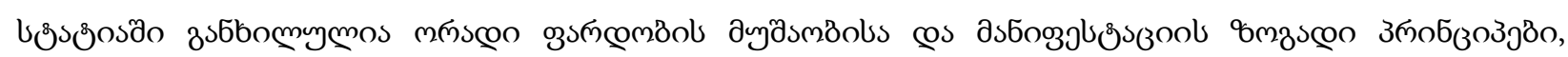

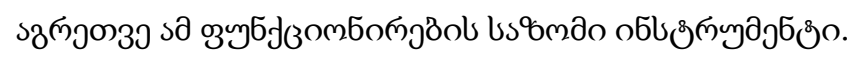

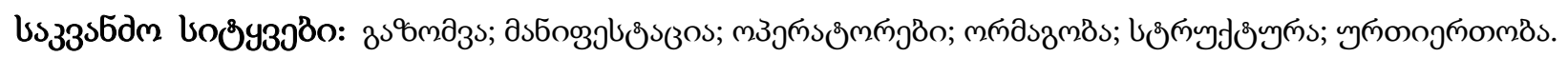

ISSN 1512-0996

www.shromebi.gtu.ge 


\section{UDC 513.72}

SCOPUS CODE 1802

https://doi.org/10.36073/1512-0996-2021-1-106-118

\section{Динамика двойственных отношений}

Серго Дадунашвили Департамент электротехники и электроники, Грузинский технический университет, Грузия, 0160, Тбилиси, ул. М. Костава 75

E-mail: dadu@gtu.ge

\section{Рецензенты:}

Г. Дгебуадзе, профессор факультета энергетики и телекоммуникации, ГТУ

E-mail: project7@gtu.ge

К. Камкамидзе, профессор факультета информатики и компьютерных систем, ГТУ

E-mail: kkamkamidze@yahoo.com

Аннотация. Природа создаёт свои системы в виде последовательности уровней иерархии, которые объединяют множества разнородных объектов, на основе двойственных отношений.

В статье рассматриваются общие принципы работы и проявления двойственных отношений, а также измерительный инструмент их функционирования.

Ключевые слова: двойственность; измерение; операторы; отношения; проявление; структура.

The date of review 13.11.2020

The date of submission 14.12.2020

Signed for publishing 29.03.2021 